\title{
UNBOUNDED HERMITIAN OPERATORS ON KOLASKI SPACES
}

\author{
JAMES JAMISON AND RAENA KING \\ Department of Mathematical Sciences, The University of Memphis, Memphis, TN 38152, USA \\ e-mail: jjamison@memphis.edu; rbryant1@memphis.edu
}

(Received 20 December 2012; revised 23 May 2013; accepted 28 May 2013; first published online 30 August 2013)

\begin{abstract}
We investigate strongly continuous one-parameter $\left(C_{0}\right)$ groups of isometries acting on certain spaces of analytical functions which were introduced by Kolaski (C. J. Kolaski, Isometries of some smooth normed spaces of analytic functions, Complex Var. Theory Appl. 10(2-3) (1988), 115-122). We characterize the generators of these groups of isometries and also the spectrum of the generators. We provide an example on the Bloch space of an unbounded hermitian operator with non-compact resolvent.
\end{abstract}

2010 AMS Classifications. 46, 47.

1. Introduction. An operator $\mathrm{A}$ on a Banach space $\mathrm{X}$ is called hermitian if $i A$ is the generator of a one-parameter $\left(C_{0}\right)$ group of isometries. Uniformly continuous groups have bounded generators, while strongly continuous groups have unbounded generators (cf. [3]). The generators of uniformly continuous $\left(C_{0}\right)$ groups are the bounded hermitian operators studied by Vidav [12]. Berkson and Porta in [1] showed that the bounded hermitian operators on $H^{p}$ for $p \neq 2$ are just real multiples of the identity. In this paper we study one-parameter groups of surjective isometries on spaces, which were first introduced by Novinger and Oberlin [10] and studied later in more generality by Kolaski [8]. These spaces, which we call Kolaski spaces, consist of analytical functions on the disk with derivative in one of the classical spaces of analytical function spaces such as $H^{p}$. In this paper we determine the generators of certain $\left(C_{0}\right)$ groups of isometries and describe their spectrum. We then provide an application of our results to the $S^{p}$ spaces, which are special cases of a Kolaski space. Since the spaces studied are classical spaces of analytical functions on the disk, the wellknown theorems on isometries of these spaces involve automorphisms of the disk. The groups of surjective isometries of these spaces are classified by the fixed point structure of the associated group of disk automorphisms.

In the first two sections we focus on the strongly continuous $\left(C_{0}\right)$ groups of isometries on a general Kolaski space, determining the corresponding generator and its spectrum. In the third section we specify the Kolaski space by requiring that the derivative of the functions belong to a classical Hardy space. Using the results of Berkson and Porta [1], we give a more complete description of the spectrum of the generator. In the last section of the paper we provide an interesting example which shows that the fixed point structure of the groups of disk automorphisms can have a strong effect on the spectral properties of the generator. 
2. $\left(C_{0}\right)$ groups of Linear Operators on $S_{N}$. Kolaski [8] introduced a special class of Banach spaces of analytical functions as follows. Let $H(D)$ be the space of analytical functions on the unit disk such that $N(f)<\infty$, where $N: H_{N} \rightarrow[0, \infty)$ is a norm, and let $S_{N}$ denote the space of functions such that $N\left(f^{\prime}\right)<\infty$. We will denote the norm of a function $f$ on $H_{N}$ with $\|f\|_{H_{N}}$ and the norm of a function $g$ on $S_{N}$ with $\|g\|_{S_{N}}$, where the norm on $S_{N}$ is given by $\|g\|_{S_{N}}=|g(0)|+\left\|g^{\prime}\right\|_{H_{N}}$. We recall that a Banach space is called smooth if its norm is weakly differentiable at every point except the origin (see [9]). Throughout this paper $H_{N}$ will always be a smooth space.

Kolaski [8] characterized the surjective linear isometries of $S_{N}$ as follows.

THEOREM 2.1. [8, Theorem 1]. Let $T$ be an isometry of $S_{N}$ onto respectively (into) $S_{N}$. Then there is a linear isometry $\mathcal{T}$ of $H_{N}$ onto respectively (into) $H_{N}$ and a $\lambda$ with $|\lambda|=1$ such that

$$
T f(z)=\lambda\left[f(0)+\int_{0}^{z} \mathcal{T} f^{\prime}(\xi) d \xi\right]
$$

We use this theorem to study the one-parameter $\left(C_{0}\right)$ groups on $S_{N}$ and determine the respective generators. We follow standard terminology as used in [3, p. 614]. A family $\left\{T_{t}\right\} t \in \mathbb{R}$ of bounded linear operators in a Banach space $\mathrm{X}$ is called a strongly continuous group if

(i) $T_{s+t}=T_{s} T_{t}$,

(ii) $T_{0}=I$,

(iii) for each $x \in X$, the map $t \rightarrow T_{t} x$ is continuous as a function of $t \in \mathbb{R}$.

These groups are called $\left(C_{0}\right)$ groups if $\lim _{t \rightarrow 0} T_{t} f=f$ for every $f \in X$. If $\left\{T_{t}\right\}$ is any $\left(C_{0}\right)$ group of linear operators, then the generator $A$ of $\left\{T_{t}\right\}$ is defined by

$$
\text { (*) } A f=\lim _{t \rightarrow 0} \frac{T_{t} f-f}{t}
$$

where the domain of $\mathrm{A}, D(A)$, is the set of all $f \in X$ for which the limit $(*)$ exists. The group $\left\{T_{t}\right\}$ is uniformly continuous if and only if its generator is a bounded linear operator A. If $\left\{T_{t}\right\}$ is strongly continuous, but not uniformly continuous, then its generator is an unbounded operator (cf. [3]).

We first consider $\left(C_{0}\right)$ groups of surjective isometries on the space $S_{N}$ and determine the generator of such a group.

Proposition 2.2. Let $\left\{T_{t}\right\}$ be a $\left(C_{0}\right)$ group of surjective linear isometries on $S_{N}$. Then there is a one-parameter group of unimodular complex numbers $\left\{\lambda_{t}\right\}$ and a $\left(C_{0}\right)$ group of surjective linear isometries $\left\{\mathcal{T}_{t}\right\}$ on $H_{N}$, such that

$$
T_{t} f(z)=\lambda_{t}\left[f(0)+\int_{0}^{z} \mathcal{T}_{t} f^{\prime}(\xi) d \xi\right]
$$

Proof. From Kolaski's theorem (2.1), a one-parameter group of surjective linear isometries on $S_{N}, T_{t}$ is given by $\left(T_{t} f\right)(z)=\lambda_{t}\left[f(0)+\int_{0}^{z} \mathcal{T}_{t} f^{\prime}(\xi) d \xi\right]$, where for each $t, \mathcal{T}_{t}$ is a surjective linear isometry of $H_{N}$ and $\lambda_{t}$ is a unimodular constant. It can be shown 
by using constant functions that $T_{t}$ induces a $\left(C_{0}\right)$ group of unimodular scalars, $\lambda_{t}$, of the form $\lambda_{t}=e^{i \gamma t}$ and hence

$$
T_{t}=e^{i \gamma t}\left[f(0)+\int_{0}^{z} \mathcal{T}_{t} f^{\prime}(\xi) d \xi\right] .
$$

The property that $T_{0}=I$ gives $e^{i \gamma(0)}\left[f(0)+\int_{0}^{z} \mathcal{T}_{0} f^{\prime}(\xi) d \xi\right]=f(z)$. Differentiating this last equation we get $\mathcal{T}_{0}=I$. Likewise, the property that $T_{s}\left(T_{t}\right)=T_{s+t}$ gives $e^{i \gamma s} e^{i \gamma t}\left[f(0)+\int_{0}^{z} \mathcal{T}_{s}\left(\mathcal{T}_{t} f\right)^{\prime}(\xi) d \xi\right]=e^{i \gamma(s+t)}\left[f(0)+\int_{0}^{z} \mathcal{T}_{s+t} f^{\prime}(\xi) d \xi\right]$. Again differentiating this last equation gives $\mathcal{T}_{s}\left(\mathcal{T}_{t}\right)=\mathcal{T}_{s+t}$. Since $T_{t}$ is strongly continuous, and

$$
\left\|\left(T_{t}-I\right) f\right\|_{S_{N}}=\left\|\left(\mathcal{T}_{t}-I\right) f^{\prime}\right\|_{H_{N}}+\left|\left(e^{i \gamma t}-1\right) f(0)\right| \geq\left\|\left(\mathcal{T}_{t}-I\right) f^{\prime}\right\|_{H_{N}},
$$

it follows that $\mathcal{T}_{t}$ is strongly continuous.

Proposition 2.3. Let $\mathcal{S}$ be the generator of the $\left(C_{0}\right)$ group $\left\{T_{t}\right\}$ on $S_{N}$, let $\mathcal{R}$ be the generator of the $\left(C_{0}\right)$ group of isometries $\left\{\mathcal{T}_{t}\right\}$ on $H_{N}$ induced by $\left\{T_{t}\right\}$ and $e^{i \gamma t}$ is a $\left(C_{0}\right)$ group of unimodular complex numbers. Then $D(\mathcal{S})=\left\{f \in S_{N}: f^{\prime} \in D(\mathcal{R})\right\}$. Further, $\mathcal{S} f(z)=i \gamma f(0)+\int_{0}^{z} \mathcal{R} f^{\prime}(\xi) d \xi$.

Proof. We will show that

$$
\begin{aligned}
& \lim _{t \rightarrow 0}\left\|\frac{T_{t} f-f}{t}-\left(i \gamma f(0)+\int_{0}^{z} \mathcal{R}\left(f^{\prime}\right)(\xi) d \xi\right)\right\|_{S_{N}}=0, \\
& \left\|\frac{T_{t} f-f}{t}-i \gamma f(0)-\int_{0}^{z} \mathcal{R} f^{\prime}(\xi) d \xi\right\|_{S_{N}} \\
& =\left\|\left[\frac{e^{i \gamma t} f(0)+e^{i \gamma t} \int_{0}^{z} \mathcal{T}_{t} f^{\prime}(\xi) d(\xi)-f(z)}{t}\right]-i \gamma f(0)-\int_{0}^{z} \mathcal{R} f^{\prime}(\xi) d \xi\right\|_{S_{N}} \\
& =\|\left[\frac{e^{i \gamma t} f(0)+e^{i \gamma t} \int_{0}^{z}\left(\mathcal{T}_{t} f^{\prime}(\xi)-f^{\prime}(\xi)\right) d \xi+e^{i \gamma t} f(z)-e^{i \gamma t} f(0)-f(z)}{t}\right] \\
& -i \gamma f(0)-\int_{0}^{z} \mathcal{R} f^{\prime}(\xi) d \xi \|_{S_{N}} \\
& \leq\left\|e^{i \gamma t}\left[\frac{\left(\mathcal{T}_{t} f^{\prime}(z)-f^{\prime}(z)\right)}{t}-\mathcal{R} f^{\prime}(z)\right]\right\|_{H_{N}}+\left\|\mathcal{R} f^{\prime}(z)\left(e^{i \gamma t}-1\right)\right\|_{H_{N}} \\
& +\left|\left(\frac{\left(e^{i \gamma t}-1\right)}{t}-i \gamma\right) f(0)\right| \text {. }
\end{aligned}
$$

Clearly, the right side $\rightarrow 0$ as $t \rightarrow 0$ since $\mathcal{R}$ is the generator of the group $\left\{\mathcal{T}_{t}\right\}$ and $e^{i \gamma t}-1 \rightarrow 0$ as $t \rightarrow 0$.

3. Spectrum of the generator of $\left\{T_{t}\right\}$. We recall that the spectrum of an operator $\mathcal{S}$ on a Banach space $X$, denoted by $\sigma(\mathcal{S})$, is the set of all complex numbers $\mu$ such 
that $\mathcal{S}-\mu I$ is non-invertible on $X$. The complement of this set is the resolvent and is denoted by $\rho(\mathcal{S})$. The point spectrum of $\mathcal{S}, \sigma_{p}(S)$, is the set of eigenvalues of $\mathcal{S}$. Hille and Phillips [6, p. 210] showed that if a generator of a one-parameter group has non-empty, compact resolvent, then it has pure point spectrum. We show how the spectrum of the generator of a $\left(C_{0}\right)$ group on $H_{N}$ is related to the generator of the $\left(C_{0}\right)$ group on $S_{N}$. In this section, following is our main result: Then

THEOREM 3.1. Let $\left\{T_{t}\right\}$ be a strongly continuous $\left(C_{0}\right)$ group of isometries on $S_{N}$.

$$
T_{t}=e^{i \gamma t}\left[f(0)+\int_{0}^{z} \mathcal{T}_{t} f^{\prime}(\xi) d \xi\right],
$$

where $\left\{\mathcal{T}_{t}\right\}$ is a strongly continuous $\left(C_{0}\right)$ group of isometries on $H_{N}$. Let $\mathcal{R}$ be the generator of $\left\{\mathcal{T}_{t}\right\}$. Let $\mathcal{S}$ be the generator of $\left\{T_{t}\right\}$. If $\mathcal{R}$ has compact resolvent, then $\mathcal{S}$ has compact resolvent.

We begin by showing the relationship between the point spectrums for the generators of groups on $H_{N}$ and $S_{N}$.

PROPOSITION 3.2. Let $\left\{T_{t}=e^{i \gamma t}\left[f(0)+\int_{0}^{z} \mathcal{T}_{t} f^{\prime}(\xi) d \xi\right]\right\}$ be a strongly continuous $\left(C_{0}\right)$ group of isometries on $S_{N}$ with generator $S$, where $\left\{\mathcal{T}_{t}\right\}$ is a strongly continuous $\left(C_{0}\right)$ group of isometries on $H_{N}$ with generator $\mathcal{R}$. Then $\sigma_{p}(\mathcal{R}) \subseteq \sigma_{p}(\mathcal{S})$ or $\sigma_{p}(\mathcal{S}) \backslash\{i \gamma\} \subseteq \sigma_{p}(\mathcal{R})$.

Proof. Let $\mu$ be an eigenvalue of $\mathcal{R}$. So $\exists f_{\mu} \in H_{N}$ such that $\mathcal{R} f_{\mu}(z)=\mu f_{\mu}(z)$. Define the function $f(z)=\int_{0}^{z} f_{\mu}(\xi) d \xi$. Then $\mathcal{S} f(z)=i \gamma f(0)+\int_{0}^{z} \mathcal{R} f^{\prime}(\xi) d \xi=\int_{0}^{z} \mathcal{R} f_{\mu}(\xi) d \xi=$ $\mu \int_{0}^{z} f_{\mu}(\xi) d \xi=\mu f(z)$, so $\mu$ is an eigenvalue of $\mathcal{S}$.

Let $\mu$ be an eigenvalue of $\mathcal{S}$. So $\exists f_{\mu} \in S_{N}$ such that $\mathcal{S} f_{\mu}(z)=\mu f_{\mu}(z)$. We first suppose that $f_{\mu}$ is not constant. Then $i \gamma f_{\mu}(0)+\int_{0}^{z} \mathcal{R} f^{\prime}(\xi) d \xi=\mu f_{\mu}$. Differentiation yields that $\mathcal{R} f_{\mu}^{\prime}(z)=\mu f_{\mu}^{\prime}$, so $\mu$ is an eigenvalue of $\mathcal{S}$. If $f_{\mu}$ is constant then $\mathcal{S} f_{\mu}=i \gamma f_{\mu}$ and so $\mu=i \gamma$.

To discover properties of the generator of $\left\{T_{t}\right\}$, it is advantageous to consider $S_{N}$ as the $\ell_{1}$ direct sum of the spaces $\mathbb{C}$ and $H_{N}$. Define the map $V: S_{N} \rightarrow \mathbb{C} \oplus_{1} H_{N}$ by $V f(z)=\left(f(0), f^{\prime}\right)$. It is easy to check that $S_{N}$ is isometric to $\mathbb{C} \oplus_{1} H_{N}$, since the norm of $\mathbb{C} \oplus_{1} H_{N}$ is given by $\|(\alpha, g)\|_{*}=|\alpha|+\|g\|_{H_{N}}$. Moreover, $V^{-1}(\alpha, g)=\alpha+\int_{0}^{z} g^{\prime}(\xi) d \xi$.

Proposition 3.3. Let $\left\{T_{t}\right\}$ be a one-parameter $\left(C_{0}\right)$ group of isometries on $S_{N}$. Define $V_{t}=V T_{t} V^{-1}$, with $V f=\left(f(0), f^{\prime}\right)$. Then $\left\{V_{t}\right\}$ is a $\left(C_{0}\right)$ group of isometries on $\mathbb{C} \oplus_{1} H_{N}$.

Proof. We will show that $\left\{V_{t}\right\}$ has the properties of a $\left(C_{0}\right)$ group of isometries. Clearly, $\left\{V_{t}\right\}$ is an isometry for each $t$.

Also, $V_{0}=V T_{0} V^{-1}=V I V^{-1}=I$ and $V_{s} V_{t}=\left(V T_{s} V^{-1}\right)\left(V T_{t} V^{-1}\right)=V T_{s} T_{t} V^{-1}=$ $V_{s+t}$.

Furthermore,

$$
\begin{aligned}
\left\|V_{t}(\alpha, g)-(\alpha, g)\right\|_{*} & =\left\|V T_{t} V^{-1}(\alpha, g)-(\alpha, g)\right\|_{*} \\
& =\left\|V T_{t}\left(\alpha+\int_{0}^{z} g^{\prime}(\xi) d \xi\right)-(\alpha, g)\right\|_{*} \\
& =\| V\left(\lambda_{t}\left(\alpha+\int_{0}^{z} \mathcal{T}_{t} g^{\prime}(\xi) d \xi\right)-(\alpha, g) \|_{*}\right. \\
& =\left\|\left(\lambda_{t}, \mathcal{T}_{t} g\right)-(\alpha, g)\right\|_{*} .
\end{aligned}
$$


Clearly, each term on the right side of this last equation goes to 0 as $t \rightarrow 0$. Hence, $\left\{V_{t}\right\}$ is strongly continuous and this completes the proof.

For the remainder of this section, we will assume that $H_{N}$ admits only trivial bounded hermitian operators. This is the case for the $H^{p}$ spaces for $p \neq 2$. An application of a theorem by Fleming and Jamison [4, Theorem 2.5, p 174] gives that if the factor spaces of an $\ell_{1}$ sum have have only trivial hermitian operators then the surjective isometries are diagonal. So $V_{t}=\left(\begin{array}{cc}\lambda_{t} & 0 \\ 0 & \mathcal{T}_{t}\end{array}\right)$.

Proposition 3.4. Let $\left\{T_{t}\right\}$ be a one-parameter $\left(C_{0}\right)$ group of isometries on $S_{N}$ and suppose that $H_{N}$ admits only trivial hermitian operators. Then by Proposition (2.2), $\left\{T_{t}\right\}$ induces $\left(C_{0}\right)$ groups $\left\{\lambda_{t}=e^{i \gamma t}\right\}$ and $\left\{\mathcal{T}_{t}\right\}$ on $\mathbb{C}$ and $H_{N}$ respectively. Let $\gamma$ be the generator of $\left\{\lambda_{t}\right\}$ and let $\mathcal{R}$ be the generator of $\left\{\mathcal{T}_{t}\right\}$. Then $\mathcal{G}=\left(\begin{array}{ll}\gamma & 0 \\ 0 & \mathcal{R}\end{array}\right)$ is the generator of the induced group $\left\{V_{t}\right\}$ on $\mathbb{C} \oplus_{1} H_{N}$.

Proof.

$$
\begin{aligned}
\left\|\left(\begin{array}{ll}
\gamma & 0 \\
0 & \mathcal{R}
\end{array}\right)\left(\begin{array}{l}
\alpha \\
g
\end{array}\right) p-\frac{1}{t}\left(V_{t}\left(\begin{array}{l}
\alpha \\
g
\end{array}\right)-\left(\begin{array}{l}
\alpha \\
g
\end{array}\right)\right)\right\|_{*} & =\left\|\left(\begin{array}{l}
\gamma \alpha \\
\mathcal{R} g
\end{array}\right)-\frac{1}{t}\left(\left(\begin{array}{cc}
\lambda_{t} & 0 \\
0 & \mathcal{T}_{t}
\end{array}\right)\left(\begin{array}{l}
\alpha \\
g
\end{array}\right)-\left(\begin{array}{l}
\alpha \\
g
\end{array}\right)\right)\right\|_{*} \\
& =\left\|\left(\begin{array}{c}
\gamma \alpha \\
\mathcal{R} g
\end{array}\right)-\frac{1}{t}\left(\left(\begin{array}{c}
\lambda_{t} \alpha \\
\mathcal{T}_{t} g
\end{array}\right)-\left(\begin{array}{l}
\alpha \\
g
\end{array}\right)\right)\right\|_{*} \\
& =\left\|\left(\begin{array}{c}
\gamma \alpha \\
\mathcal{R} g
\end{array}\right)-\frac{1}{t}\left(\begin{array}{c}
\lambda_{t} \alpha-\alpha \\
\mathcal{T}_{t} g-g
\end{array}\right)\right\|_{*} .
\end{aligned}
$$

Thus, $\lim _{t \rightarrow 0}\left\|\left(\begin{array}{c}\gamma \alpha \\ \mathcal{R} g\end{array}\right)-\frac{1}{t}\left(V_{t}\left(\begin{array}{l}\alpha \\ g\end{array}\right)-\left(\begin{array}{l}\alpha \\ g\end{array}\right)\right)\right\|_{*}=\lim _{t \rightarrow 0}\left(\left|\gamma \alpha-\frac{\lambda_{t} \alpha-\alpha}{t}\right|+\left\|\mathcal{R} g-\frac{\mathcal{T}_{t} g-g}{t}\right\|_{H_{N}}\right)=$ 0 , since $\gamma$ and $\mathcal{R}$ are generators of $\left\{\lambda_{t}\right\}$ and $\left\{\mathcal{T}_{t}\right\}$ respectively. This completes the proof.

Proposition 3.5. Let $\left\{T_{t}\right\}$ be a one-parameter $\left(C_{0}\right)$ group of isometries on $S_{N}$ and $\left\{\lambda_{t}\right\}$ and $\left\{\mathcal{T}_{t}\right\}$ be the induced groups on $\mathbb{C}$ and $H_{N}$ respectively. Let $\mathcal{R}$ be the generator of $\left\{\mathcal{T}_{t}\right\}$ and $\lambda_{t}=e^{i \gamma t}$. Let $\mathcal{G}=\left(\begin{array}{ll}\gamma & 0 \\ 0 & \mathcal{R}\end{array}\right)$ be the generator of the induced group $\left\{V_{t}\right\}$ on $\mathbb{C} \oplus_{1} H_{N}$. If $\mu \neq \gamma$ is not an eigenvalue of $\mathcal{R}$ and $\mathcal{R}-\mu I$ is surjective, then $\mathcal{G}-\mu I$ is surjective.

Proof. Given $(\alpha, g) \in \mathbb{C} \oplus_{1} H_{N}$, we want to show that there exists $(z, h) \in \mathbb{C} \oplus_{1}$ $H_{N}$ such that $(\mathcal{G}-\mu I)(z, h)=(\alpha, g)$ Let $(\alpha, g) \in \mathbb{C} \oplus_{1} H_{N}$. Consider $(\mathcal{G}-\mu I)\left(\begin{array}{l}z \\ h\end{array}\right)=$ $\left(\begin{array}{cc}\gamma-\mu & 0 \\ 0 & (\mathcal{R}-\mu I)\end{array}\right)\left(\begin{array}{l}z \\ h\end{array}\right)=\left(\begin{array}{c}(\gamma-\mu) z \\ (\mathcal{R}-\mu I) h\end{array}\right)=\left(\begin{array}{l}\alpha \\ g\end{array}\right)$. The surjectivity of $(\mathcal{R}-\mu I)$ and $\gamma \neq \mu$ implies that $\left(\begin{array}{l}\alpha \\ g\end{array}\right)$ is in the range of $(\mathcal{G}-\mu I)$. This completes the proof.

Proposition 3.6. Let $\left\{T_{t}\right\}$ be a one-parameter $\left(C_{0}\right)$ group of isometries on $S_{N}$ and $\left\{\lambda_{t}\right\}$ and $\left\{\mathcal{T}_{t}\right\}$ be the induced groups on $\mathbb{C}$ and $H_{N}$ respectively. Let $\mathcal{R}$ be the generator of $\left\{\mathcal{T}_{t}\right\}$ and $\lambda_{t}=e^{i \gamma t}$. Let $\mathcal{G}=\left(\begin{array}{ll}\gamma & 0 \\ 0 & \mathcal{R}\end{array}\right)$ be the generator of the induced group $\left\{V_{t}\right\}$ on $\mathbb{C} \oplus_{1} H_{N}$. If $\mathcal{R}$ has compact resolvent, then $\mathcal{G}$ has compact resolvent. 
Proof. Let $(\mathcal{G}-\mu I)^{-1}\left(\begin{array}{l}z_{n} \\ g_{n}\end{array}\right)$ be a bounded sequence in the resolvent of $\mathcal{G}$.

$$
(\mathcal{G}-\mu I)^{-1}\left(\begin{array}{c}
z_{n} \\
g_{n}
\end{array}\right)=\left(\begin{array}{cc}
\frac{1}{\gamma-\mu} & 0 \\
0 & (\mathcal{R}-\mu I)^{-1}
\end{array}\right)\left(\begin{array}{l}
z_{n} \\
g_{n}
\end{array}\right)=\left(\begin{array}{c}
\frac{z_{n}}{\gamma-\mu} \\
(\mathcal{R}-\mu I)^{-1} g_{n}
\end{array}\right) .
$$

Since $\frac{z_{n}}{\gamma-\mu}$ is a bounded sequence of complex numbers, it has a convergent subsequence, say $\frac{z_{n_{k}}}{\gamma-\mu}$. Furthermore, since $g_{n}$ is a bounded sequence of $H_{N}$ functions and $(\mathcal{R}-\mu I)^{-1}$ is compact on $H_{N},(\mathcal{R}-\mu I)^{-1} g_{n_{k}}$ is a bounded sequence, so it has a convergent subsequence, say $(\mathcal{R}-\mu I)^{-1} g_{n_{k_{i}}}$. Thus, $(\mathcal{G}-\mu I)^{-1}\left(\begin{array}{l}z_{n} \\ g_{n}\end{array}\right)$ has a convergent subsequence, and the resolvent of $\mathcal{G}$ is compact.

The main theorem is an immediate consequence of the preceding propositions.

4. Application to $S^{p}$ spaces. In this section we consider a special example of a Kolaski space, namely $S^{p}$. This Banach space consists of analytical functions $f$ on the disk with $f^{\prime} \in H^{p}$. The norm is given $\|f\|=|f(0)|+\left\|f^{\prime}\right\|_{H^{p}}$. The bounded hermitian operators on this space were classified by Hornor and Jamison in [7]. In this section we will determine the generators of a certain type of strongly continuous $\left(C_{0}\right)$ groups of isometries and thereby characterize certain unbounded hermitian operators associated with these groups in terms of their action on the space. Berkson and Porta, cf. [1], determined the generator of the one-parameter $\left(C_{0}\right)$ group of isometries on $H^{p}$ and this result will be crucial to our work. Since $H^{p}$ is a smooth space for $1<p<\infty$, their result together with the previous results on the Kolaski spaces can be used to find the generator of the type (i) one-parameter $\left(C_{0}\right)$ group of isometries on $S^{p}$.

In order to apply their result, we first introduce some notation and results. Let a one-parameter group of disk automorphisms be denoted by $\left\{\phi_{t}\right\}$ with

$$
\phi_{t}(z)=\frac{a_{t}\left(z-b_{t}\right)}{1-\bar{b}_{t} z}
$$

where $a_{t}$ and $b_{t}$ are constants such that $\left|a_{t}\right|=1$ and $\left|b_{t}\right|<1$. A non-constant oneparameter $\left(C_{0}\right)$ group of disk automorphisms $\left\{\phi_{t}\right\}$ on D is said to be of type (i) if the set of common fixed points in the extended complex plane of functions $\phi_{t}$ is a doubleton subset consisting of a point $\tau \in D$ and $\bar{\tau}^{-1}\left(\bar{\tau}^{-1}=\infty\right.$ if $\left.\tau=0\right)$. We will call a one-parameter $\left(C_{0}\right)$ group, $\left\{T_{t}\right\}$, on $S^{p}$ of type (i) if the induced one-parameter $\left(C_{0}\right)$ group of disk automorphisms is of type (i). To state our results we will quote the following result by Berkson and Porta [1] on the form of the one-parameter $\left(C_{0}\right)$ group of disk automorphisms.

THEOREM 4.1. [1, Theorem 1.10]. Let $\left\{\phi_{t}\right\}, t \in \mathbb{R}$ be a non-constant one-parameter group of disk automorphisms of D such that $\phi_{t}(z)$ is continuous in t for each $z \in D$. If $\left\{\phi_{t}\right\}$ is of type ( $i$ ), then there are uniquely determined (by $\left\{\phi_{t}\right\}$ ) constants $\tau$ and $c$, the former in $D$ and the latter real and non-zero such that the parameters $a_{t}$ and $b_{t}$ of equation (4) are given for all $t \in \mathbb{R}$ by

$$
a_{t}=\frac{|\tau|^{2}-e^{i c t}}{|\tau|^{2} e^{i c t}-1} ; \quad b_{t}=\frac{\tau\left(e^{i c t}-1\right)}{e^{i c t}-|\tau|^{2}} .
$$


The constant $\tau$ is, in fact, the unique element of $D$ which is left fixed by all $\phi_{t}$ for $t \in \mathbb{R}$. The constant $c$ satisfies and is determined by the equation $e^{i c t} z=\left(\Psi \circ \phi_{t} \circ \Psi\right)(z)$ for $t \in \mathbb{R}$ and $z \in D$ and $\Psi$ a disk automorphism given by

$$
\Psi(z)=\frac{z-\tau}{\bar{\tau} z-1} .
$$

Conversely, if $\tau$ is any element of $D$ and $c$ is any non-zero real number, the parameters $a_{t}$ and $b_{t}$, defined for each $t \in \mathbb{R}$ as in (5) have moduli satisfying the requirements in (4), and defined by the formula in (4) a one-parameter group, $\left\{\phi_{t}\right\}$ of type (i).

Associated with every continuous one-parameter group of disk automorphisms there is the so-called invariance polynomial. It was shown in [1] that the zeroes of this polynomial are precisely the set of fixed points of $\phi_{t}$ in the finite plane.

COROLlary 4.2. [1, Corollary 1.13]. If $\left\{\phi_{t}\right\}$ is a group of type (i), then its invariance polynomial can be written in the form

$$
R(z)=\frac{-i c\left(\bar{\tau} z^{2}-\left(1+|\tau|^{2}\right) z+\tau\right)}{1-|\tau|^{2}}
$$

for all $z \in \mathbb{C}$, where $\tau$ is the unique point of $D$ fixed by every $\phi_{t}$ and $c$ is the unique constant as in Theorem 4.1.

We also recall the form of the surjective isometries on $H^{p}$ for $1 \leq p<\infty, p \neq 2$, which were determined by Forelli [5].

Proposition 4.3. [5, Theorem 2]. If $A$ is a linear isometry of $H^{p}$ onto $H^{p}, 1 \leq p \leq \infty$, $p \neq 2$, then there is a disk automorphism $\phi$ and $\lambda \in \mathbb{C}$ such that

$$
(A f)(z)=\lambda\left[\phi^{\prime}(z)\right]^{1 / p} f(\phi(z))
$$

for all $f \in H^{p}$ and $z \in D$.

On the other hand, if $1 \leq p \leq \infty$ and $\lambda$ is a unimodular constant and $\phi$ is a disk automorphism, then (8) defines a linear isometry of $\mathrm{H}^{p}$ onto $\mathrm{H}^{p}$.

Let $\left\{T_{t}\right\}$ be a one-parameter $\left(C_{0}\right)$ group of isometries induced by a group of disk automorphisms of type (i) and define a hermitian operator $A$ to be of type (i) if it is the generator of a group of isometries where the associated group of disk automorphisms is of type (i). Berkson and Porta [1] defined $\mathcal{R}$ with the domain, $\mathcal{D}(\mathcal{R})$, to be all functions $f \in H^{p}$ such that the function

$$
\mathcal{R}=R f^{\prime}+(1 / p) R^{\prime} f
$$

is in $H^{p}$, where $R$ is the invariance polynomial given in (7) and juxtaposition indicates multiplication. They proved the following theorem.

THEOREM 4.4. [1, Theorem 3.1]. If $A$ is a hermitian operator of type (i) in $H^{p}$, $1 \leq p<\infty, p \neq 2$, then:

(1) there is a unique real number $\beta$ such that $A=\beta I-i \mathcal{R}$, where $\mathcal{R}$ is as in (9);

(2) the eigenvalues of $A$ are precisely the real numbers $\sigma_{n}=c(n+1 / p)+\beta, n=$ $0,1,2, \ldots$; 
(3) for each $n$, the eigenmanifold of $A$ corresponding to $\sigma_{n}$ is the one-dimensional span of the function $f_{n, \tau}(z)$, where for each $z \in D$

$$
f_{n, \tau}(z)= \begin{cases}(z-\tau)^{n} /\left(z-\bar{\tau}^{-1}\right)^{n+(2 / p)} & \text {,if } \tau \neq 0 \\ z^{n} \quad, \text { if } \tau=0 & \end{cases}
$$

(4) A has compact resolvent, and hence pure point spectrum.

We now combine this theorem of Berkson and Porta [1] along with one of the main results of this paper, Corollary (3.1), to find the generator and spectrum of the associated one-parameter $\left(C_{0}\right)$ group of isometries on $S^{p}$.

COROLlARY 4.5. Let $\mathcal{S}$ be the generator of type $(i)$ of a one-parameter $\left(C_{0}\right)$ group of isometries on $S^{p}$. Then $\mathcal{D}(\mathcal{S})=\left\{f \in S^{p}: f^{\prime} \in \mathcal{D}(\mathcal{R})\right\}$, where $\mathcal{R}$ is given in (9) and

$$
\mathcal{S} f(z)=\gamma f(0)+\int_{0}^{z} \beta f^{\prime}(\xi)-\left(\mathcal{R} f^{\prime}\right)(\xi) d \xi=(\gamma-\beta) f(0)+\beta f(z)-\int_{0}^{z}\left(\mathcal{R} f^{\prime}\right)(\xi) d \xi,
$$

where $\gamma$ and $\beta$ are generators of one-parameter $\left(C_{0}\right)$ groups of complex numbers and $\mathcal{R}$ is as given in (9). Furthermore, $\mathcal{S}$ has compact resolvent, thus pure point spectrum, so

$$
\sigma(\mathcal{S})=\left\{\sigma_{n}=c(n+(1 / p))+\beta: n=0,1,2, \ldots\right\},
$$

where the eigenmanifolds corresponding to each $\sigma_{n}$ are spanned by the functions

$$
g_{n, \tau}= \begin{cases}\int_{0}^{z}(\xi-\tau)^{n} /\left(\xi-\bar{\tau}^{-1}\right)^{n+(2 / p)} d \xi & \text { if } \tau=0 \\ (n+1)^{-1} z^{n+1} & \text { if } \tau \neq 0\end{cases}
$$

Proof. The proof is a direct application of our results from Sections 1 and 2 for $S_{N}$ and $H_{N}$ by considering $H_{N}=H^{p}$.

REMARK 4.6. The first two terms in $\mathcal{S}$ gives the form of the bounded hermitian operators on $S^{p}$, cf. [7].

5. An example in the Bloch Space. In the previous section we assumed that $H_{N}$ is a smooth Banach space. We now consider an example of a $\left(C_{0}\right)$ group of isometries on a non-smooth Banach space. Rather than considering the associated Kolaski space, we directly focus on the $S_{N}$ group, since the $\left(C_{0}\right)$ group properties on $H_{N}$ are reflected in $S_{N}$. We give an example of a one-parameter $\left(C_{0}\right)$ group of isometries in which the properties of the generator in the preceding sections do not hold and in fact the resolvent is not compact. The space we wish to consider is the Bloch space and is defined as follows:

$$
\mathcal{B}=\left\{f: \mathrm{f} \text { is analytical on } D, f(0)=0 \text {, and } \sup _{|z|<1}\left|f^{\prime}(z)\right|\left(1-|z|^{2}\right)=N(f)<\infty\right\} \text {. }
$$

Let $\mathcal{B}_{0}$ denote the closed subspace of $\mathcal{B}$ spanned by all polynomials. Cima and Wogen [2] characterized the surjective isometries of $\mathcal{B}_{0}$ as follows. 
LEMMA 5.1. [2, Corollary 3]. If $S: \mathcal{B}_{0} \rightarrow \mathcal{B}_{0}$ is a surjective isometry, then there is a disk automorphism $\phi$ of $D$ and $\lambda$ such that $|\lambda|=1$ so that

$$
S f(z)=\lambda[f(\phi(z))-f(\phi(0))] \quad \forall f \in \mathcal{B}_{0} .
$$

We show an example of a $\left(C_{0}\right)$ one-parameter group in the case where $\phi_{t}$ is as in equation (4) with $a_{t}=1$ and $b_{t}=\left(1-e^{-t}\right)\left(1+e^{t}\right)^{-1}$. Hence,

$$
\phi_{t}(z)=\frac{\left(1+e^{-t}\right) z+\left(1-e^{-t}\right)}{\left(1-e^{-t}\right) z+\left(1+e^{-t}\right)} .
$$

In this case the generator of $\phi_{t}$ is given by $R=\left(1-z^{2}\right) / 2$ and $\left.\frac{\partial}{\partial t}\right|_{t=0} \phi_{t}^{\prime}=R^{\prime}=-z$. Furthermore, $\phi_{t}$ is a one-parameter $\left(C_{0}\right)$ group of disk automorphisms.

For each $t \in \mathbb{R}, T_{t} f(z)=\lambda_{t}\left[f\left(\phi_{t}(z)\right)-f\left(\phi_{t}(0)\right)\right]$. With the given $\phi_{t}$, if $\left\{T_{t}\right\}$ is a oneparameter $\left(C_{0}\right)$ group of isometries on $\mathcal{B}_{0}$, then it is easy to show that $\left\{\lambda_{t}\right\}$ is a $\left(C_{0}\right)$ group of unimodular complex numbers.

THEOREM 5.2. Let $\left\{\phi_{t}\right\}$ be given as in equation (10) and $\beta$ be the generator of $\left\{\lambda_{t}\right\}$. Then $\mathcal{G} f(z)=i \beta f(z)+R f^{\prime}(z)$ is the generator of $\left\{T_{t}\right\}$, where $\mathcal{R}=\left(1-z^{2}\right) / 2$.

Proof. We show that $\lim _{t \rightarrow 0}\left\|\frac{T_{t} f-f}{t}-\mathcal{G}(f)\right\|=0$ for every $f \in \mathcal{D}(\mathcal{G})$.

$$
\begin{aligned}
&\left\|\frac{T_{t} f-f}{t}-\mathcal{G}(f)\right\| \\
&=\left\|\frac{e^{i \beta t}\left[f \circ \phi_{t}-f \circ \phi_{t}(0)\right]-f}{t}-\left(i \beta f+\left(1-z^{2}\right) f^{\prime} / 2\right)\right\| \\
&=\sup _{|z|<1}\left(1-|z|^{2}\right)\left[\left\|\frac{e^{i \beta t}\left(f^{\prime} \circ \phi_{t}\right)\left(\phi_{t}^{\prime}\right)-f^{\prime}}{t}-i \beta f^{\prime}-\left(1-z^{2}\right) f^{\prime \prime} / 2-\left(-z f^{\prime}\right)\right\|\right] \\
& \leq \sup _{|z|<1}\left(1-|z|^{2}\right)\left(\left\|\frac{e^{i \beta t}\left(f^{\prime} \circ \phi_{t}\right)\left(\phi_{t}^{\prime}\right)-e^{i \beta t} \phi_{t}^{\prime} f^{\prime}}{t}-\left(1-z^{2}\right) f^{\prime \prime} / 2\right\|\right. \\
&\left.+\left\|\frac{e^{i \beta t} \phi_{t}^{\prime} f^{\prime}-\phi_{t}^{\prime} f^{\prime}}{t}-i \beta f^{\prime}\right\|+\left\|\frac{\phi_{t}^{\prime} f^{\prime}-f^{\prime}}{t}-\left(-z f^{\prime}\right)\right\|\right) \\
&= \sup _{|z|<1}\left(1-|z|^{2}\right)\left\{\left\|\frac{e^{i \beta t} \phi_{t}^{\prime}\left(f^{\prime} \circ \phi_{t}-f^{\prime} \circ \phi_{0}\right)}{\phi_{t}-\phi_{0}} \cdot \frac{\phi_{t}-\phi_{0}}{t}-\left(1-z^{2}\right) f^{\prime \prime} / 2\right\| \|\right. \\
&\left.+\left\|\frac{\phi_{t}^{\prime} f^{\prime}\left(e^{i \beta t}-1\right)}{t}-i \beta f^{\prime}\right\|+\left\|\frac{\phi_{t}^{\prime}-\phi_{0}^{\prime}}{t}-(-z)^{\prime} f^{\prime}\right\|\right\} .
\end{aligned}
$$

Each term of the previous equation goes to 0 as $t \rightarrow 0$. Thus, the proof is complete.

We now find the point spectrum of $\mathcal{G}$.

COROLLARY 5.3. Let $\mathcal{G}$ be the generator of the one-parameter $\left(C_{0}\right)$ group of isometries $\left\{T_{t}\right\}$ on $\mathcal{B}_{0}$ in the previous theorem induced by the group of disk automorphisms in (10). Then $\sigma_{p}(\mathcal{G})=\emptyset$. 
Proof. We find values of $\alpha$ for which the differential equation $\mathcal{G} f=\alpha f$ has a solution. Then,

$$
\left(1-z^{2}\right) f^{\prime} / 2+i \beta f=\alpha f
$$

After rearranging and integrating, the equation becomes

$$
\int\left(f^{\prime} / f\right) d z=2(\alpha-i \beta) \int\left(1-z^{2}\right)^{-1} d z
$$

Hence, $\log f=(\alpha-i \beta)(\log (1+z)-\log (1-z))+C$. Whence, $f(z)=\left(\frac{1+z}{1-z}\right)^{\alpha-i \beta} e^{C}$. However, $f(0) \neq 0$, so the solution is not in $\mathcal{B}_{0}$. Therefore, $\sigma_{p}(\mathcal{G})=\emptyset$.

To find a representation for $(\mathcal{G}-\lambda I)^{-1}$, we solve the differential equation $(\mathcal{G}-$ $\lambda I) f(z)=g(z)$ for $g$ by using an integrating factor of $\left[(1+z)(1-z)^{-1}\right]^{i \beta-\lambda}$. Thus,

$$
(\mathcal{G}-\lambda I)^{-1} g(z)=\left[(1-z)(1+z)^{-1}\right]^{i \beta-\lambda} \int_{0}^{z} 2\left(1-w^{2}\right)^{-1}\left[(1+w)(1-w)^{-1}\right]^{i \beta-\lambda} g(w) d w .
$$

Before giving our last result, we recall that a closed operator with non-empty resolvent set and compact resolvent always has only eigenvalues in its spectrum [6, Theorem 56.14.2].

COROLlARY 5.4. Let $\mathcal{G}$ be the generator of a one-parameter $\left(C_{0}\right)$ group of isometries on $\mathcal{B}_{0}$ induced by the group of disk automorphisms in (10). Then the resolvent of $\mathcal{G}$ is not compact.

Proof. To prove this, we only need to show that the resolvent of $\mathcal{G}$ in non-empty. Palmer notes that if $i \mathcal{G}$ is the infinitesimal generator of a one-parameter group of isometries, then $\sigma(\mathcal{G}) \subset \mathbb{R}$ (see [11, p. 387]). Thus, the resolvent of $\mathcal{G}$ is non-empty.

We leave open the question concerning the form of the unbounded hermitian operators on $\mathcal{B}_{0}$ induced by other groups of disk automorphisms.

\section{REFERENCES}

1. E. Berkson and H. Porta, Hermitian operators and one-parameter groups of isometries in Hardy spaces, Trans. Amer. Math. Soc. 185 (1973), 331-344.

2. J. A. Cima and W. R. Wogen, On isometries of the Bloch space, Illinois J. Math. 24 (2) (1980), 313-316.

3. N. Dunford and J. T. Schwartz, Linear operators, part I (Interscience, New York, 1958)

4. R. J. Fleming and J. E. Jamison, Hermitian operators and isometries on sums of Banach spaces, Proc. Edinburgh Math. Soc. 32 (2) (1989), 169-191.

5. F. Forelli, The isometries of Hp, Can. J. Math. 16 (1964), 721-728.

6. E. Hille and R. S. Phillips, Functional analysis and semi-groups, American Mathematical Society Colloquium Publications, vol. 31 (American Mathematical Society, Providence, RI, 1957).

7. W. Hornor and J. E. Jamison, Isometries of some Banach spaces of analytic functions, Integral Equ. Operator Theory 41 (4) (2001), 410-425.

8. C. J. Kolaski, Isometries of some smooth normed spaces of analytic functions, Complex Var. Theory Appl. 10 (2-3) (1988), 115-122.

9. S. Li and S. Stevic, Products of integral-type operators between Bloch spaces. J. Math. Anal. Appl. 349 (2009), 596-610. 
10. W. P. Novinger and D. M. Oberlin, Linear isometries of some normed spaces of analytic functions, Can. J. Math. 37 (1) (1985), 62-74.

11. T. W. Palmer, Unbounded normal operators on Banach spaces, Trans. Amer. Math. Soc. 133 (1968), 385-414.

12. I. Vidav, Eine metrische Kennzeichnung der selbstadjungierten Operatoren, Math. Z. 66 (1956), 121-128. 